\title{
基于量子生成对抗网络的信息隐藏方案
}

\author{
罗佳 ${ }^{1,2}$, 周日贵 ${ }^{1,2)^{*}}$, 李尧肿 ${ }^{1,2}$, 刘广钟 ${ }^{1)}$ \\ 1) (上海海事大学信息工程学院 上海 201306) \\ 2) (智能信息处理与量子智能计算研究中心 上海 201306) \\ (rgzhou@shmtu.edu.cn)
}

\begin{abstract}
摘 要: 针对量子图像信息隐藏技术在面对基于统计的隐写分析算法检测时的不安全问题，提出基于量子生成对抗 网络(QGAN)的信息隐藏方案. 首先利用映射规则将秘密信息映射为单量子比特门, 演化得到 QGAN 生成器 $G$ 的参 数化量子电路的输人态; 然后通过 QGAN 中的生成电路生成含密量子图像; 最后通过测量含密量子图像得到样本数 据后与真实数据样本作为判别器 $D$ 的输人进行迭代优化, 使 $G$ 能够得到接近于目标图像的含密图像. 实验结果表 明, 所提方案能生成与目标图像分布较好拟合的含密图像, 完成信息的非嵌人式隐藏.
\end{abstract}

关键词：量子生成对抗网络; 信息隐藏; 量子图像隐写; 量子图像

中图法分类号: TP391.41

DOI: $10.3724 /$ SP.J.1089.2021.18617

\section{Information Hiding Scheme Based on Quantum Generative Adversarial Network}

\author{
Luo Jia $^{1,2)}$, Zhou Rigui ${ }^{1,2)^{*}}$, Li Yaochong ${ }^{1,2)}$, and Liu Guangzhong ${ }^{1)}$ \\ 1) (College of Information Engineering, Shanghai Maritime University, Shanghai 201306) \\ ${ }^{2)}$ (Research Center of Intelligent Information Processing and Quantum Intelligent Computing, Shanghai 201306)
}

\begin{abstract}
Due to the insecurity of quantum image information hiding technology in the face of statistical-based steganalysis algorithm detection, an information hiding scheme based on quantum generative adversarial network (QGAN) is proposed. This scheme first uses the mapping rules to map the secret information into the single qubit gate to prepare for the input state of the parameterized quantum circuit of the generator $G$. Then the stego quantum image is generated by the generating circuit in QGAN. Finally, the sample data obtained by measuring the stego image and the real data are used as the input of the discriminator $D$. The iterative optimization is performed so that $G$ can obtain a stego image close to the target image. The experimental results show that proposed scheme can generate stego images that fit the target image distribution well and achieve the non-embedded hiding of information.
\end{abstract}

Key words: QGAN; information hiding; quantum image steganography; quantum image

随着云计算、大数据及物联网等现代互联网技 术的发展，信息技术早已融人人们的日常生活之 中. 个人信息、商业信息乃至国家的机密信息等都 被存储于互联网，其中很多信息能够被大众和企
业使用，从而形成了便捷的网络化生活. 然而，信 息技术的广泛应用在给大众的生活和工作带来便 捷的同时，也暴露出很多突出的信息安全问题. 如 何保证各类信息的内容安全，防止信息在通信的

收稿日期：2020-08-31; 修回日期：2021-04-07. 基金项目：国家重点研发计划重点专项(2018YFC1200200, 2018YFC1200205). 罗佳(1995一), 男, 博士研究生, 主要研究方向为量子图像处理、量子信息隐藏; 周日贵(1973一), 男, 博士, 教授, 博士生导师, 论文 通讯作者, 主要研究方向为量子图像处理、量子机器学习; 李尧羽(1996-), 男, 博士研究生, 主要研究方向为量子机器学习; 刘广钟 (1962-), 男, 博士, 教授, 博士生导师, 主要研究方向为智能信息处理、水声信号处理与通信、高性能网络. 
过程中被窃听、截获、破坏和篡改，成为信息安全 领域的重要研究问题. 信息隐藏技术通过某种方 法把秘密信息隐藏于公开信息之中，并且不影响 公开信息的正常传播与使用, 从而很好地保护了 秘密信息的安全. 此外，近些年量子信息技术相关 研究领域得到了快速的发展, 量子信息越来越多 地以一种大容量、安全的信息载体形式出现. 因此, 学者们通过借鉴经典信息隐藏的思想和方法，将 研究目光投向了量子信息隐藏技术, 即量子信道 下的新型信息安全保护技术.

量子图像信息隐藏是量子信息隐藏技术的一 大分支, 最早在 2008 年, Mogos ${ }^{[1]}$ 提出一种利用 3 级量子比特表示图像像素值的量子信息隐藏协议, 成功地将经典图像信息隐藏的思想扩展到量子信 息领域. 如今常用的量子图像信息隐藏方法是基 于嵌人的方法，通过修改载体图像将秘密消息嵌人 图像的视觉冗余部分隐藏信息, 主要有空域 ${ }^{[2-3]}$ 、变 换域 ${ }^{[4-6]}$ 等信息隐藏方法. 这类量子信息隐藏技术 的基础是将经典图像存储在量子态中，其中，灵活 量子图像表示(flexible representation of quantum images，FRQI) ${ }^{[7]}$ 和新型增强量子表示 (novel enhanced quantum representation, NEQR $)^{[8]}$ 的方法使 用最为广泛. 2012 年, Iliyasu 等 ${ }^{[2]}$ 基于 FRQI 和受限 的几何变换提出一种量子水印及认证协议. 之后, Zhang 等 ${ }^{[3]}$ 提出一种基于 FRQI 方法的量子傅里叶 变换水印协议, 将秘密信息嵌人在量子图像的傅 里叶变换系数中, 通过傅里叶变换的性质保证了 嵌人的水印能够抵御噪声和剪切攻击. 2016 年, Jiang 等 ${ }^{[4]}$ 提出基于 NEQR 方法的最低有效位量子 图像隐写算法. 2019 年, 王兵等 ${ }^{[5]}$ 提出基于量子索 引图像的隐写算法, 利用量子数据矩阵和量子调 色板矩阵的索引嵌人信息.

尽管有许多的基于空域和变换域的量子图像 信息隐藏算法问世，但是现有的算法都是基于嵌 人的方法, 会在载体图像上留下一定程度的修改 痕迹而很难抵抗隐写分析方法的检测 ${ }^{[9]}$. 在经典 信息隐藏领域，为了抵抗隐写分析方法的检测, 文献[10-13]提出各类基于机器学习的无载体信息 隐藏方法，旨在通过秘密消息直接生成或得到含 密图像而不需要修改载体图像，从而在根本上抵 抗隐写分析方法的检测.

近些年, 由于寻求通过量子计算解决机器学 习的运算效率问题，因此出现了越来越多结合量 子计算和机器学习的研究. 因此, 量子生成对抗网 络(quantum generative adversarial networks, QGAN)
受到了相关学者的关注, 其也在理论上显示出了 与经典版本相比具有指数加速的潜力 ${ }^{[14]}$. 这些研 究中有些是通过训练量子生成器创建一个表示数 据潜在概率分布的量子状态 ${ }^{[15-17]}$ ，有些是根据训 练数据的目标分布生成经典数据样本 ${ }^{[18-20]}$

本文旨在借助 QGAN 的图像生成能力, 设计 一个基于 QGAN 的信息隐藏方案. 该方案首先以 目标图像概率分布作为目标分布进行训练, 随后 以秘密信息间接作为量子生成器的输人生成量子 载体图像，从而实现信息隐藏的目的.

\section{1 相关工作}

\section{1 泡利矩阵}

众所周知，处理量子比特的基本方式是量子 逻辑门. 使用量子逻辑门, 有目的地使量子态发生 演化. 泡利矩阵就是单量子比特量子态演化的重 要组成部分, 其所表示的单量子比特 $\boldsymbol{I}, \boldsymbol{X}, \boldsymbol{Y}, \boldsymbol{Z}$ 门 代表着对量子态矢量最基本的操作, 它们的矩阵 形式为

$$
\left\{\begin{array}{l}
\boldsymbol{\sigma}_{0} \equiv \boldsymbol{I} \equiv\left[\begin{array}{ll}
0 & 1 \\
1 & 0
\end{array}\right] \\
\boldsymbol{\sigma}_{1} \equiv \boldsymbol{\sigma}_{x} \equiv \boldsymbol{X} \equiv\left[\begin{array}{ll}
0 & 1 \\
1 & 0
\end{array}\right] \\
\boldsymbol{\sigma}_{2} \equiv \boldsymbol{\sigma}_{y} \equiv \boldsymbol{Y} \equiv\left[\begin{array}{rr}
0 & -\mathrm{i} \\
\mathrm{i} & 0
\end{array}\right] \\
\boldsymbol{\sigma}_{3} \equiv \boldsymbol{\sigma}_{z} \equiv \boldsymbol{Z} \equiv\left[\begin{array}{rr}
1 & 0 \\
0 & -1
\end{array}\right]
\end{array}\right.
$$

并且对于任意的单量子比特进行操作的西变换 $U$, 存在实数 $a, b, c$ 使 $U=\mathrm{e}^{-\mathrm{i} \theta\left(a \sigma_{x}+b \sigma_{y}+c \sigma_{z}\right)}$.

此外, 单量子比特旋转门 $\boldsymbol{R}_{x}, \boldsymbol{R}_{y}, \boldsymbol{R}_{z}$ 也是通过 不同的泡利矩阵作为生成元构成的. 它们的矩阵 形式为

$$
\left\{\begin{array}{l}
\boldsymbol{R}_{x}(\theta)=\mathrm{e}^{-\mathrm{i} \theta \sigma_{x} / 2}=\left(\begin{array}{rr}
\cos (\theta / 2) & -\mathrm{i} \sin (\theta / 2) \\
-\mathrm{i} \sin (\theta / 2) & \cos (\theta / 2)
\end{array}\right) \\
\boldsymbol{R}_{y}(\theta)=\mathrm{e}^{-\mathrm{i} \theta \sigma_{y} / 2}=\left(\begin{array}{rr}
\cos (\theta / 2) & -\sin (\theta / 2) \\
\sin (\theta / 2) & \cos (\theta / 2)
\end{array}\right) . \\
\boldsymbol{R}_{z}(\theta)=\mathrm{e}^{-\mathrm{i} \theta \sigma_{z} / 2}=\left(\begin{array}{rr}
\mathrm{e}^{-\mathrm{i} \theta / 2} & 0 \\
0 & \mathrm{e}^{\mathrm{i} \theta / 2}
\end{array}\right)
\end{array}\right.
$$

\section{2 新型增强量子表示}

NEQR 模型使用 2 个纠缠的量子比特序列存 储颜色和位置信息, 并将整个图像存储到 2 个量子 比特序列的叠加中. 对于二值图像, NEQR 模型将 
颜色信息存储到一个量子比特中。一幅 $2^{n} \times 2^{n}$ 的 二值图像可以表示为

$$
|\boldsymbol{I}\rangle=\frac{1}{2^{n}} \sum_{h=0}^{2^{n}-1} \sum_{w=0}^{2^{n}-1}\left|i_{h w}\right\rangle|h\rangle|w\rangle .
$$

其中, $|h\rangle|w\rangle$ 表示图像的坐标信息; $\left|i_{h w}\right\rangle$ 表示对应 坐标的颜色信息.

\section{3 经典生成对抗网络}

生成对抗网络(generative adversarial networks, $\mathrm{GAN})^{[21]}$ 是一个二人零和博弯过程, 生成器 $G$ 生成 样本以模拟真实数据的分布欺骗判别器 $D, D$ 判 别目标是真实数据还是生成样本, 其训练过程如 图 1 所示.

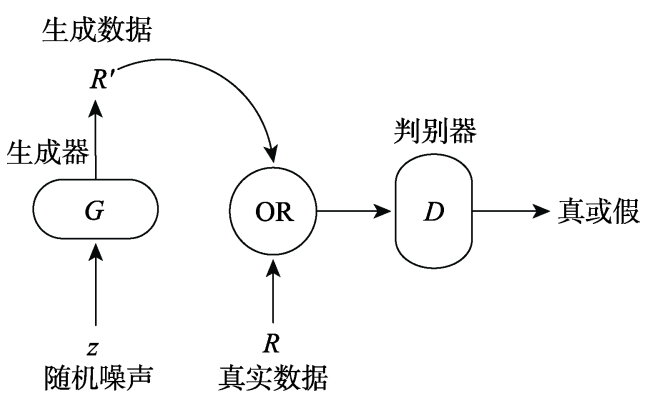

图 1 经典 GAN 框架

首先 $G$ 将随机噪声作为输人产生生成样本, 随后 $D$ 收到从真实数据里抽样的样本或 $G$ 产生的 样本, 然后输出一个值判断输人的样本是来自真 实数据还是 $G$ 的输出. 这 2 个过程相互对抗并迭代 优化, 使 $D$ 和 $G$ 的性能不断提升, 最终使 $G$ 与 $D$ 二者之间达到纳什平衡. 将这一过程数学形式化, 得到一个最小最大化问题

$$
\begin{gathered}
\min _{G} \max _{D}\left(E_{x \sim p_{\text {real }}(x)}[\ln D(x)]+\right. \\
\left.E_{z \sim p_{\text {noise }}(z)}[\ln (1-D(G(z)))]\right) .
\end{gathered}
$$

其中， $D(x)$ 表示 $x$ 是真实图像的概率; $G(z)$ 表示 从输人噪声 $z$ 产生的生成图像.

因为 $G$ 和 $D$ 都是由可微的神经网络来表示, 那么就可以用随机梯度下降法优化模型的参数. 在实际的训练过程中, 需要同时对 $G$ 和 $D$ 的参数 进行优化. 也就是说, 每次迭代分别根据 $G$ 和 $D$ 的损失函数对各自参数进行优化.

\section{2 本文方案}

\subsection{QGAN}

根据判别器和生成器的模型和其数据源是经
典的还是量子的区别, QGAN 的模型也有许多的不 同. 本文采用混合 $\mathrm{QGAN}$, 其生成器 $G_{\theta}$ 是通过参 数化变分量子电路实现的, 而判别器 $D_{\phi}$ 采用的依 然是经典的神经网络. 其总体结构如图 2 所示.

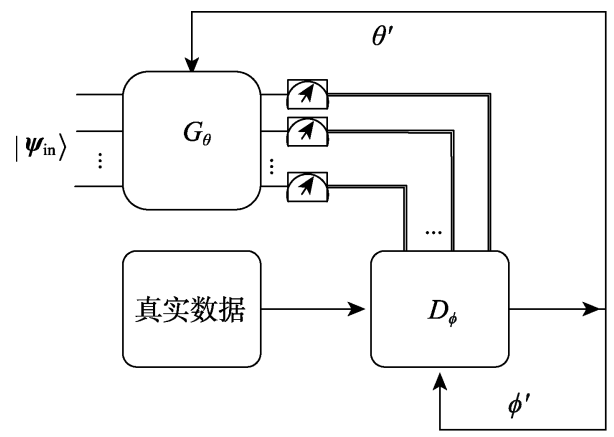

图 2 QGAN 框架

给定一个 $N$ 量子比特的输人态 $\left|\boldsymbol{\psi}_{\text {in }}\right\rangle$, 对于电 路参数为 $\theta$ 的 $G_{\theta}$, 其输出态为

$$
\left|\boldsymbol{g}_{\theta}\right\rangle=G_{\theta}\left|\boldsymbol{\psi}_{\text {in }}\right\rangle=\sum_{j=0}^{2^{N}-1} \sqrt{p_{\theta}^{j}}|j\rangle .
$$

其中, $p_{\theta}^{j}$ 表示相应量子态所测得的概率, 其满足 量子态归一化条件.

如图 3 所示, 本文所采用的 $G_{\theta}$ 具有与文献 [16]相似的量子电路架构, 电路是由 $L$ 层西变换操 作 $U_{l}$ 和最后的测量层构成. 而电路中每层西变换 操作都由 2 部分组成, 分别是单量子比特旋转层和 两量子比特纠缠层. 本文所使用的单量子比特旋 转门为泡利 $Y$ 旋转门 $\left(\boldsymbol{R}_{y}\right)$, 两量子比特纠缠门使 用的是控制 $Z$ 门(controlled- $Z, C Z$ ). 单量子比特 旋转层形式化为 $\boldsymbol{R}_{y}\left(\theta_{i}^{l}\right)$, 其中 $l$ 为层的索引, 范围 为 $1 \sim L ; i$ 为量子比特的索引, 范围为 $0 \sim N-1$. 为了纠缠所有量子比特, 本文采用了无参数的控 制 $Z$ 门，其矩阵表示为

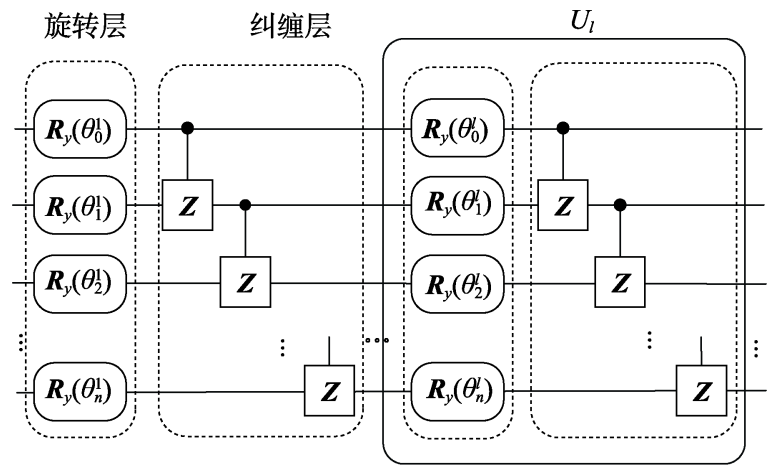

图 3 量子生成电路 


$$
\mathbf{C Z}=\left(\begin{array}{rrrr}
1 & 0 & 0 & 0 \\
0 & 1 & 0 & 0 \\
0 & 0 & 1 & 0 \\
0 & 0 & 0 & -1
\end{array}\right) .
$$

可以看到, 每层中所有参数都在单量子比特 旋转层中, 所以整个 $G_{\theta}$ 电路总的参数个数为 $\mathrm{NL}$.

由于 $G_{\theta}$ 生成的样本需要经过测量得到结果, 也就是说, $D_{\phi}$ 的输人都是经典数据. 因此, 本文 采用的 $D_{\phi}$ 是一个经典的神经网络, 由一层输人 层、一层隐藏层以及一层输出层组成. 输出层的值 $D_{\phi}(x)$ 为 $[0,1]$. 当 $D_{\phi}(x)=0$ 时, 表示 $D_{\phi}$ 判定输人 样本是 $G_{\theta}$ 生成的样本; 当 $D_{\phi}(x)=1$ 时, 表示 $D_{\phi}$ 判 定输人样本来自真实数据. 即, $G_{\theta}$ 的目的就是使 自身生成的样本能够骗过 $D_{\phi}$, 使输出值 $D_{\phi}(x)$ 等 于 1. 因此, $G_{\theta}$ 和 $D_{\phi}$ 形成了一个相互对抗的过程. 此外，本文 $G_{\theta}$ 和 $D_{\phi}$ 的损失函数分别定义为

$$
L_{G_{\theta}}=-E_{x \sim p_{\theta}}\left[\ln D_{\phi}(x)\right] \text {; }
$$

$L_{D_{\phi}}=-\frac{1}{2}\left(E_{x \sim p_{\text {real }}}\left[\ln D_{\phi}(x)\right]+E_{x \sim p_{\theta}}\left[\ln \left(1-D_{\phi}(x)\right)\right]\right)$.

其中, $p_{\theta}$ 和 $p_{\text {real }}$ 分别表示 $G_{\theta}$ 的输出分布和真实数 据分布. 在对抗训练的过程中，同时使用随机梯度 下降法来优化这 2 个损失函数. 对于 $D_{\phi}$ 的损失函 数对参数的梯度, 使用经典的优化算法即可轻易 地求出. 而对于 $G_{\theta}$ 的梯度表示为

$$
\nabla_{\theta} L_{G_{\theta}}=-\sum_{x} \ln D_{\phi}(x) \frac{\partial P_{\theta}(x)}{\partial \theta} .
$$

因为本文是通过投影测量得到的服从输出概 率分布的样本 $x$, 而非直接得到输出概率分布表 达式 $p_{\theta}(x)$, 所以无法直接计算 $G_{\theta}$ 的梯度.

但是从参数化量子电路角度考虑, $\frac{\partial P_{\theta}(x)}{\partial \theta}$ 可 以写成

$$
\frac{\partial P_{\theta}(x)}{\partial \theta}=\frac{\partial}{\partial \theta}\left\langle x\left|U_{k: 1} \rho_{0} U_{k: 1}^{\dagger}\right| x\right\rangle
$$

其中， $\boldsymbol{\rho}_{0}$ 表示电路输人的初始态的密度矩阵; $U_{k: 1}$ 表示电路的 $k$ 个西变换, 即 $U_{k: 1}=U_{k} U_{k-1} \cdots U_{1}$. 假 设参数 $\theta$ 只存在于第 $j$ 个西变换 $U_{j}$ 中, 那么式(1) 的偏导可以转换成

$$
\begin{aligned}
& \frac{\partial P_{\theta}(x)}{\partial \theta}=\left\langle x\left|U_{k: j+1} \frac{\partial U_{j}}{\partial \theta} U_{j-1: 1} \rho_{\theta} U_{k: 1}^{\dagger}\right| x\right\rangle+ \\
& \left\langle x\left|U_{k: 1} \rho_{0} U_{j-1: 1}^{\dagger} \frac{\partial U_{j}^{\dagger}}{\partial \theta} U_{k: j+1}^{\dagger}\right| x\right\rangle
\end{aligned}
$$

而量子电路中的西变换操作即量子门可以通 过泡利矩阵生成, 也就是对于每个西变换都有 $U_{j}(\theta)=\exp \left(-\mathrm{i} \theta_{j} P_{j} / 2\right)$. 因此, 将 $\frac{\partial U_{j}(\theta)}{\partial \theta}=-\frac{\mathrm{i}}{2} U_{j} P_{j}$ 和 $\frac{\partial U_{j}^{\dagger}(\theta)}{\partial \theta}=\frac{\mathrm{i}}{2} P_{j} U_{j}^{\dagger}$ 代人式(2)可以得到

$$
\begin{aligned}
& \frac{\partial P_{\theta}(x)}{\partial \theta}=-\frac{\mathrm{i}}{2}\left\langle x\left|U_{k: j+1} U_{j} P_{j} U_{j-1: 1} \rho_{0} U_{k: 1}^{\dagger}\right| x\right\rangle+ \\
& \frac{\mathrm{i}}{2}\left\langle x\left|U_{k: 1} \rho_{0} U_{j-1: 1}^{\dagger} P_{j} U_{j}^{\dagger} U_{k: j+1}^{\dagger}\right| x\right\rangle= \\
& -\frac{\mathrm{i}}{2}\left\langle x\left|U_{k: j+1} U_{j} P_{j} \underline{U_{j-1: 1} \rho_{0} U_{j-1: 1}^{\dagger}} U_{k: j}^{\dagger}\right| x\right\rangle+ \\
& \frac{\mathrm{i}}{2}\left\langle x\left|U_{k: j} \underline{U_{j-1: 1} \rho_{0} U_{j-1: 1}^{\dagger} P_{j} U_{j}^{\dagger} U_{k: j+1}^{\dagger}}\right| x\right\rangle= \\
& -\frac{\mathrm{i}}{2}\left\langle x\left|U_{k: j+1} U_{j}\left[P_{j}, \underline{U_{j-1: 1} \rho_{0} U_{j-1: 1}^{\dagger}}\right] U_{k: j}^{\dagger}\right| x\right\rangle
\end{aligned}
$$

因此，无法直接求对易子，但是对于任意密度 矩阵有

$$
\begin{gathered}
{\left[P_{j}, \rho\right]=\mathrm{i}\left[U_{j}\left(\frac{\pi}{2}\right) \rho U_{j}^{\dagger}\left(\frac{\pi}{2}\right)-\right.} \\
\left.U_{j}\left(-\frac{\pi}{2}\right) \rho U_{j}^{\dagger}\left(-\frac{\pi}{2}\right)\right]
\end{gathered}
$$

将式(4)代人式(3), 可以得到

$$
\begin{gathered}
\frac{\partial P_{\theta}(x)}{\partial \theta}=\frac{1}{2}\langle x| U_{k: j+1} U_{j}\left(\theta+\frac{\pi}{2}\right) U_{j-1: 1} \\
\rho_{0} U_{j-1: 1}^{\dagger} U_{j}^{\dagger}\left(\theta+\frac{\pi}{2}\right) U_{k: j+1}^{\dagger}|x\rangle- \\
\langle x| U_{k: j+1} U_{j}\left(\theta-\frac{\pi}{2}\right) U_{j-1: 1} \\
\rho_{0} U_{j-1: 1}^{\dagger} U_{j}^{\dagger}\left(\theta-\frac{\pi}{2}\right) U_{k: j+1}^{\dagger}|x\rangle
\end{gathered}
$$

进而简化式(5)得到

$$
\frac{\partial P_{\theta}(x)}{\partial \theta}=\frac{1}{2}\left(P_{\theta^{+}}(x)-P_{\theta^{-}}(x)\right)
$$

其中, $\theta^{ \pm}=\theta \pm \frac{\pi}{2} \boldsymbol{e}^{j}, \boldsymbol{e}^{j}$ 表示参数空间的第 $j$ 个单 位向量.

由式(6) 可知, 无需直接得到输出概率分布, 而是调整参数 $\theta$ 之后通过投影测量得到样本, 然 后根据式(7)求得生成电路损失函数的梯度，即

$$
\nabla_{\theta} L_{G_{\theta}}=\frac{1}{2} E_{x \sim p_{\theta}^{-}}\left[\ln D_{\phi}(x)\right]-\frac{1}{2} E_{x \sim p_{\theta}}\left[\ln D_{\phi}(x)\right]
$$

之后可以通过设定学习率，使用梯度下降法更新 参数完成优化.

\section{2 信息隐藏方案}

本文方案通过经典与量子 2 个信道协同作用 完成, 其流程如图 4 所示. 


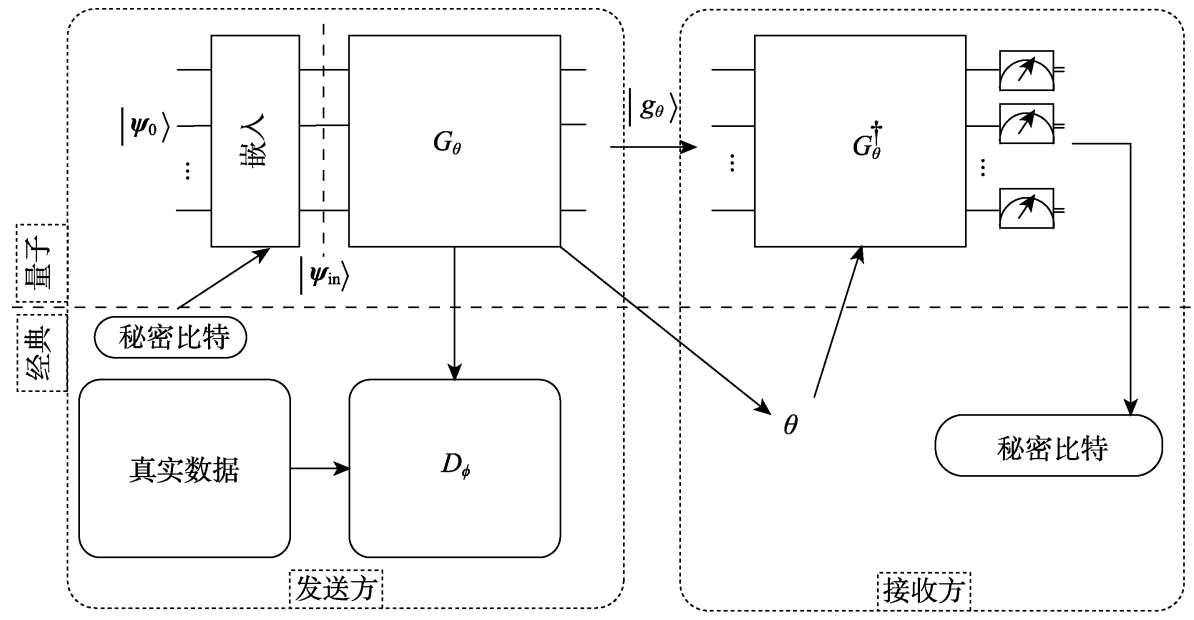

图 4 本文方案流程图

\subsection{1 秘密信息映射}

本文通过建立秘密信息比特与输人态量子比 特之间的一一映射关系，间接地将秘密信息嵌人 生成图像当中，实现了信息隐藏方案.

对于一个 $N$ 量子比特的输人态 $\left|\psi_{\text {in }}\right\rangle$, 给出原 始全 0 量子态 $\left|\psi_{0}\right\rangle=0^{\otimes N}$ ，通过将秘密信息比特映 射为作用在单量子比特上的量子门 $U$ 实现输人态 的制备. 举例来说，对于第 $j$ 个量子比特，当对应 的第 $j$ 的秘密信息比特为 0 时，此时作用在量子比 特上的门是单位门 $\boldsymbol{I}$; 当对应的秘密信息比特为 1 时, 所采用的单量子比特门为 Hadamard门 $\boldsymbol{H}$. 它 们的映射关系为

$$
U_{j}=\left\{\begin{array}{ll}
\boldsymbol{I} \equiv\left[\begin{array}{ll}
1 & 0 \\
0 & 1
\end{array}\right], & j=0 \\
\boldsymbol{H} \equiv \frac{1}{\sqrt{2}}\left[\begin{array}{cc}
1 & 1 \\
1 & -1
\end{array}\right], & j=1
\end{array} .\right.
$$

\subsection{2 秘密信息嵌人}

秘密信息的嵌人是通过将输人态 $\left|\boldsymbol{\psi}_{\text {in }}\right\rangle$ 经过 $G_{\theta}$ 参数化量子电路演化之后得到目标量子图像完 成的. 因此，首先给出目标图像

$$
|\boldsymbol{T}\rangle=\frac{1}{2^{n}} \sum_{h=0}^{2^{n}-1} \sum_{w=0}^{2^{n}-1}\left|i_{h w}\right\rangle|h\rangle|w\rangle .
$$

将其改写成与输出态概率分布相似的表示形式为

$$
|\boldsymbol{T}\rangle=\sum_{j=0}^{2^{2 n+1}-1} \sqrt{p^{j}}|j\rangle .
$$

其中, $|j\rangle=\left|i_{h w}\right\rangle|h\rangle|w\rangle ; \quad p^{j}$ 表示对应量子态所测 得的概率. 之后，通过随机初始化量子电路的参 数，训练上文所述 QGAN. 当训练完成后，得到的 生成概率分布，也就是生成图像表示为

$$
\left|\boldsymbol{g}_{\theta}\right\rangle=\sum_{j=0}^{2^{2 n+1}-1} \sqrt{p_{\theta}^{j}}|j\rangle .
$$

至此, 完成秘密信息嵌人含密图像也就是生成图 像中的过程. 此外, 还需将 $G_{\theta}$ 参数化量子电路中 的参数使用经典手段发送给接收方, 将含密图像 通过量子信道发送给接收方。

\subsection{3 秘密信息提取}

对于生成量子电路, 本文使用 $U_{G_{\theta}}$ 表示电路 对量子态的西变换. 因此, 从输人态到生成图像的 演化过程为 $\left|\boldsymbol{g}_{\theta}\right\rangle=U_{G_{\theta}}\left|\boldsymbol{\psi}_{\text {in }}\right\rangle$.

由于量子电路的可逆性质, 只需要将电路逆 置, 并设置好来自发送方的电路参数, 以生成图像 作为现输人, 即可从生成图像恢复出原始输人态 $\left|\boldsymbol{\psi}_{\text {in }}\right\rangle$, 其演化过程表示为 $\left|\boldsymbol{\psi}_{\text {in }}\right\rangle=U_{G_{\theta}}^{\dagger}\left|\boldsymbol{g}_{\theta}\right\rangle$.

在上文提出的映射规则中，当秘密信息为 1 时 采用的 $\boldsymbol{H}$ 门可以让 $|0\rangle$ 态转化为 $|0\rangle$ 态与 $|1\rangle$ 态同概 率出现, 而秘密信息为 0 时则不发生改变. 因此, 在接收方得到 $\left|\psi_{\text {in }}\right\rangle$ 后, 需要对其进行测量, 观测 其概率分布, 进而从概率分布中得到每个量子比 特所映射的秘密信息比特.

\section{3 实验结果与分析}

\section{1 实验仿真}

本文使用 Qiskit 实现基于 QGAN 的信息隐藏 方案, 并使用量子模拟对它进行验证. 因为经典计 算机上的仿真时间随量子位的数量呈指数增长, 因此将实验限制在较低数量的量子比特情况下. 本文选取了大小为 $2 \times 2$ 的 BAS(bars and stripes)图 像中的一幅作为目标图像. 即只需要 3 个量子比特 
就可以表示该图像，因此秘密信息比特也选取为 3 个比特. 该图像及其概率分布如图 5 所示.

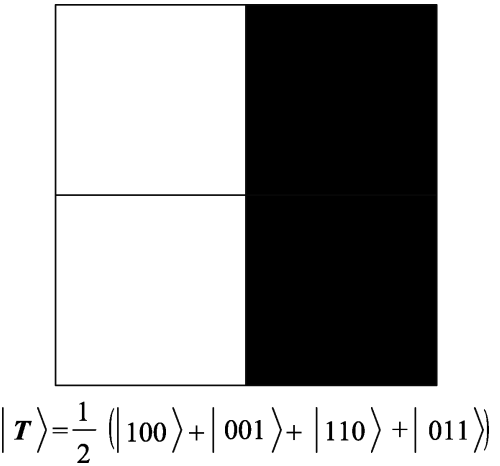

图 5 目标图像及其分布

对于 $G_{\theta}$, 本文采用的电路如图 3 所示, 深度 取为 2. 对于 $D_{\phi}$, 使用 PyTorch 实现由输人层、一 层隐藏层和输出层组成的神经网络. 输人层和隐 藏层激活函数采用 Leaky ReLU 函数, 输出层采用 Sigmoid 函数. 本文采用 AMSGRAD 优化算法 ${ }^{[22]}$, 并将初始学习率设为 $10^{-4}$.

图 6 展示当 3 个比特秘密信息选取为 111, 训 练批量为 100 时, 量子生成电路经过 3000 次对抗 训练之后, 生成图像和目标图像的概率分布. 可以 看到虽然生成的概率分布并不完全拟合理论概率 分布，但是其分布都在目标图像像素点位置且概 率相近. 这表明生成电路能够成功学习到正确的 概率分布，并生成正确的量子图像.

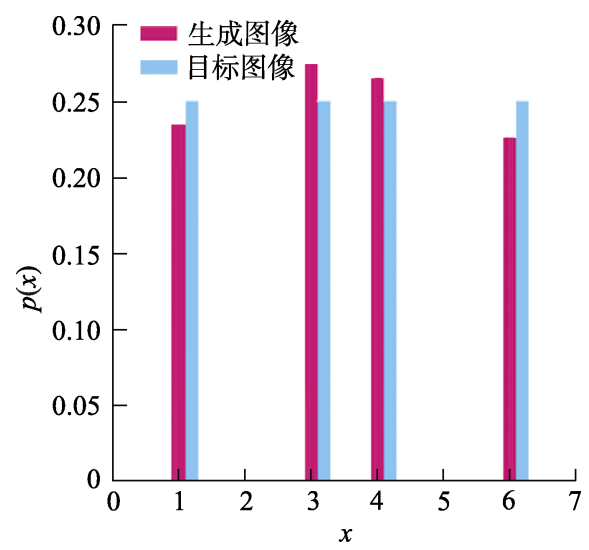

图 6 目标图像与生成图像概率分布

$\mathrm{KL}$ 散度通常用于量化 2 个概率分布之间的差 异, 对于目标图像和生成图像, 当 KL 散度的值越 趋向于 0 , 说明生成图像越接近目标图像. 图 7 给 出了随着训练的次数增加目标图像和生成图像之 间的 KL 散度的变化趋势，可以看到 KL 散度的值 随着训练渐渐趋向于 0 , 这表明本文方案的生成电 路已经能很好地生成接近目标图像的生成图像.

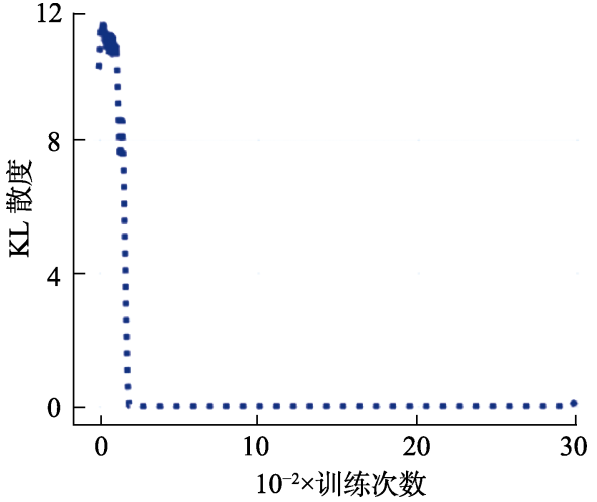

图 $7 \mathrm{KL}$ 散度

通过观察 QGAN 的损失函数, 可以更好地了 解 QGAN 的训练过程. 当训练达到对抗的均衡点 时, 也就是说 $D_{\phi}(x)=1 / 2$ 时, 代人 $G_{\theta}$ 和 $D_{\phi}$ 的损失 函数公式可得它们的损失函数都为 $-\ln 2 \approx 0.693$. 图 8 给出 $G_{\theta}$ 和 $D_{\phi}$ 的损失函数随着训练次数的变 化过程, 其中红色线表示 $G_{\theta}$ 损失函数, 蓝色线表 示 $D_{\phi}$ 损失函数. 可以看出 $G_{\theta}$ 和 $D_{\phi}$ 的损失函数都 趋近于 0.693 , 这说明 QGAN 训练是稳定、有效的.

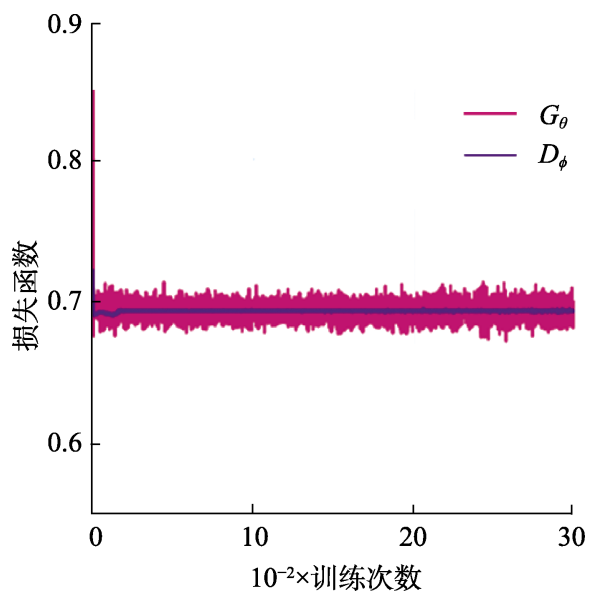

图 8 损失函数 $G_{\theta}$ 和 $D_{\phi}$ 变化过程

\section{2 嵌入容量}

与现有量子信息隐藏方案通过改变图像像素 值进行秘密信息嵌人不同, 本文在进行信息隐藏 的过程中采用的是建立秘密信息比特与作用在输 人态量子比特之间的量子门的映射关系而完成的 间接嵌人. 因此, 本文方案在每一量子比特中嵌人 一个比特的秘密信息, 即嵌人容量为 1 比特/量子 比特. 用经典参数计算嵌人容量, 也就是通过计算 所嵌人的秘密信息比特和载体图像像素个数之比, 可得对于一幅大小为 $n \times m$ 的二值图像，本文方案 的容量为

$$
C=\frac{n+m+1}{n \times m} \mathrm{bit} / \mathrm{pixel} .
$$




\section{3 安全性}

本文所研究的是基于 QGAN 的信息隐藏方案, 其安全性可以从 2 方面分析. 一方面是量子通信本 身的安全性，量子比特的传输优胜于经典通信的 地方就是量子力学中的不可克隆原理排除了对未 知量子态进行复制的可能. 也就是说, 窃听者不能 在不干扰通信的情况下复制传输的量子态. 另一 方面是本文方案自身的安全性，本文方案中隐藏 秘密信息的图像是由生成电路直接生成的与目标 图像相似的没有人为修改的伪自然图像. 所以相 比传统的量子图像信息隐藏方案，本文方案更难 引起窃听者的怀疑, 从而能更加隐蔽地进行秘密 信息传输. 此外, 即使窃听者能够直接在传输的量 子比特上进行西变换操作，但若没有只有发送方 和接收方才拥有的量子生成电路参数, 也无法提 取出秘密信息.

\section{4 电路复杂度}

量子电路的复杂度是通过计算电路中使用的 基本量子门的数量得出的, 其中基本量子门的定 义包括单量子比特门和两量子比特控制门. 为方 便与其他量子图像隐写算法比较, 假设生成图像 是大小为 $n \times n$ 、位深度为 24 的图像, 本文方案所 使用的生成网络量子电路的复杂度为 $(2 n+24+$ $2 n+23) L$. 此外, 将秘密信息映射至生成电路输人 态所需量子门数量为 $(2 n+24)$. 因此, 本文方案使 用的量子门总数为 $(4 L+2) n+47 L+24$. 由于 $L$ 是一 个较小的常数, 故电路复杂度为 $O(n)$.

表 1 给出了本文方案与文献 $[6,23]$ 信息隐藏方 案的量子电路复杂度的对比. 相比之下，本文方案 的电路复杂度具有指数级的加速. 这是因为现有 的量子图像信息隐藏方案采用的是修改载体图像 进行信息嵌人, 这一过程将经典图像制备编码为 量子态, 往往需要数量巨大的量子门. 而本文使用 变分量子电路生成含密图像的方法则克服了图像 制备过程所需的高额复杂度问题，从而取得较低 的电路复杂度.

表 1 电路复杂度对比

\begin{tabular}{cl}
\hline 信息隐藏方案 & \multicolumn{1}{c}{ 电路复杂度 } \\
\hline 文献[6] & $O\left((45 n-3) \times 2^{2 n}+2 n\right)$ \\
文献[23] & $O\left(48 n \times 2^{2 n}+3 n\right)$ \\
本文 & $O(n)$ \\
\hline
\end{tabular}

\section{4 结 语}

本文提出的基于 $\mathrm{QGAN}$ 的信息隐藏方案，以
秘密信息间接驱动生成器生成含密图像, 基本上 解决之前量子图像信息隐藏算法会在含密图像中 留有修改痕迹的问题. 该 QGAN 依赖于通过对具 有不同参数的同一量子生成器进行采样估计量子 生成器的解析梯度, 从而达到了生成与目标图像 相拟合的含密图像的目的. 通过实验分析，表明提 出的基于 QGAN 的信息隐藏方案可以有效地通过 生成简单的 BAS 图像数据分布将秘密信息进行隐 藏, 并且可以利用量子电路的可逆性原理方便地 提取出秘密信息. 此外, 通过对量子电路复杂度的 对比, 可以看出本文方案优于现有类型量子图像 信息隐藏方案. 本文首次将 QGAN 应用于信息隐 藏领域，为量子计算在更多领域的交叉应用提供 了新思路. 而如何改进秘密信息的映射方式，提高 秘密信息的隐藏容量是下一步的重点研究工作.

\section{参考文献(References):}

[1] Mogos G. Stego quantum algorithm[C] //Proceedings of the International Symposium on Computer Science and Its Applications. Los Alamitos: IEEE Computer Society Press, 2008: 187190

[2] Iliyasu A M, Le P Q, Dong F Y, et al. Watermarking and authentication of quantum images based on restricted geometric transformations[J]. Information Sciences, 2012, 186(1): 126-149

[3] Zhang W W, Gao F, Liu B, et al. A watermark strategy for quantum images based on quantum Fourier transform[J]. Quantum Information Processing, 2013, 12(2): 793-803

[4] Jiang N, Zhao N, Wang L. LSB based quantum image steganography algorithm[J]. International Journal of Theoretical Physics, 2016, 55: 107-123

[5] Wang Bing, Hao Mengqi, Li Panchi, et al. Description method and steganography of quantum indexed images[J]. Journal of Computer-Aided Design \& Computer Graphics, 2019, 31(11): 1995-2006(in Chinese)

(王兵, 郝梦奇, 李盼池, 等. 量子索引图像的描述方法与隐 写算法 [J]. 计算机辅助设计与图形学学报, 2019, 31(11): 1995-2006)

[6] Li Panchi, Zhao Ya. Quantum watermarking scheme using small-scale quantum circuits and gray-code scrambling[J]. Journal of Computer-Aided Design \& Computer Graphics, 2017, 29(9): 1624-1634(in Chinese)

(李盼池, 赵娅. 基于小规模量子线路和格雷码置乱的量子 水印方案 [J]. 计算机辅助设计与图形学学报, 2017, 29(9): 1624-1634)

[7] Le P Q, Dong F Y, Hirota K. A flexible representation of quantum images for polynomial preparation, image compression, and processing operations[J]. Quantum Information Processing, 2011, 10(1): 63-84

[8] Zhang Y, Lu K, Gao Y H, et al. NEQR: a novel enhanced quantum representation of digital images[J]. Quantum Informa- 
tion Processing, 2013, 12(8): 2833-2860

[9] Luo J, Zhou R G, Hu W W, et al. Detection of steganography in quantum grayscale images[J]. Quantum Information Processing, 2020, 19(5): Article No.149

[10] Zhou Zhili, Cao Yi, Sun Xingming. Coverless information hiding based on bag-of-words model of image[J]. Journal of Applied Sciences, 2016, 34(5): 527-536(in Chinese)

(周志立, 曹炎, 孙星明. 基于图像 Bag-of-Words 模型的无载 体信息隐藏[J]. 应用科学学报, 2016, 34(5): 527-536)

[11] Wang Yaojie, Niu Ke, Yang Xiaoyuan. Information hiding scheme based on generative adversarial network[J]. Journal of Computer Applications, 2018, 38(10): 2923-2928(in Chinese) (王耀杰，钮可，杨晓元. 基于生成对抗网络的信息隐藏方 案[J]. 计算机应用, 2018, 38(10): 2923-2928)

[12] Baluja S. Hiding images within images[J]. IEEE Transactions on Pattern Analysis and Machine Intelligence, 2019, 42(7): 1685-1697

[13] Hu D H, Wang L, Jiang W J, et al. A novel image steganography method via deep convolutional generative adversarial networks[J]. IEEE Access, 2018, 6: 38303-38314

[14] Lloyd S, Weedbrook C. Quantum generative adversarial learning[J]. Physical Review Letters, 2018, 121(4): 040502

[15] Dallaire-Demers P L, Killoran N. Quantum generative adversarial networks[J]. Physical Review A, 2018, 98(1): 012324

[16] Zoufal C, Lucchi A, Woerner S. Quantum generative adversar- ial networks for learning and loading random distributions[J]. npj Quantum Information, 2019, 5(1): Article No.103

[17] Hu L, Wu S H, Cai W Z, et al. Quantum generative adversarial learning in a superconducting quantum circuit[J]. Science Advances, 2019, 5: eaav2761

[18] He Z M, Li L Z, Zheng S G, et al. A conditional generative model based on quantum circuit and classical optimization[J]. International Journal of Theoretical Physics, 2019, 58(4): 1138-1149

[19] Situ H Z, He Z M, Wang Y Y, et al. Quantum generative adversarial network for generating discrete distribution[J]. Information Sciences, 2020, 538: 193-208

[20] Zeng J F, Wu Y F, Liu J G, et al. Learning and inference on generative adversarial quantum circuits[J]. Physical Review A, 2019, 99(5): 052306

[21] Goodfellow I J, Pouget-Abadie J, Mirza M, et al. Generative adversarial nets[C] //Proceedings of the 27th International Conference on Neural Information Processing Systems. Cambridge: MIT Press, 2014: 2672-2680

[22] Reddi S J, Kale S, Kumar S. On the convergence of Adam and beyond[OL]. [2020-08-31]. https://openreview.net/forum?id= ryQu7f-RZ

[23] Miyake S, Nakamae K. A quantum watermarking scheme using simple and small-scale quantum circuits[J]. Quantum Information Processing, 2016, 15(5): 1849-1864 